Article

\title{
Energy Analysis for the Connection of the Nuclear Reactor DEMO to the European Electrical Grid
}

\author{
Sergio Ciattaglia ${ }^{1}$, Maria Carmen Falvo ${ }^{2, * \mathbb{D}}$, Alessandro Lampasi ${ }^{3}{ }^{(1)}$ and \\ Matteo Proietti Cosimi ${ }^{2}$ \\ 1 EUROfusion Consortium, 85748 Garching, Germany; sergio.ciattaglia@euro-fusion.org \\ 2 DIAEE-Department of Astronautics, Energy and Electrical Engineering, University of Rome Sapienza, \\ 00184 Rome, Italy; m.proietticosimi@gmail.com \\ 3 ENEA Frascati, 00044 Frascati, Rome, Italy; alessandro.lampasi@enea.it \\ * Correspondence: mariacarmen.falvo@uniroma1.it
}

Received: 31 March 2020; Accepted: 22 April 2020; Published: 1 May 2020

\begin{abstract}
Towards the middle of the current century, the DEMOnstration power plant, DEMO, will start operating as the first nuclear fusion reactor capable of supplying its own loads and of providing electrical power to the European electrical grid. The presence of such a unique and peculiar facility in the European transmission system involves many issues that have to be faced in the project phase. This work represents the first study linking the operation of the nuclear fusion power plant DEMO to the actual requirements for its correct functioning as a facility connected to the power systems. In order to build this link, the present work reports the analysis of the requirements that this unconventional power-generating facility should fulfill for the proper connection and operation in the European electrical grid. Through this analysis, the study reaches its main objectives, which are the definition of the limitations of the current design choices in terms of power-generating capability and the preliminary evaluation of advantages and disadvantages that the possible configurations for the connection of the facility to the European electrical grid can have. In reference to the second objective, the work makes possible a first attempt at defining the features of the point of connection to the European grid, whose knowledge will be useful in the future, for the choice of the real construction site.
\end{abstract}

Keywords: nuclear fusion; tokamak; generation power plant; power system; electrical transmission grid

\section{Introduction}

The European roadmap to fusion energy, summarized in Figure 1, includes the DEMOnstration power plant (generally identified as DEMO), which represents the first fusion reactor designed to supply electrical power to the electrical grid to which it will be connected [1]. With the start of its operation, currently set for the middle of the 21st century, DEMO could be a revolution in the world of nuclear fusion power and in general in the world of power generation.

The first and fundamental step of the roadmap however is ITER, a research tokamak project currently under construction in France that is foreseen to be operative in around five years. ITER is not designed to generate electrical power, but it is designed to achieve five goals that are essential for the continuation of the research in this field [2]:

- It will generate $500 \mathrm{MW}$ of fusion thermal power during a relatively prolonged fusion time of $400 \mathrm{~s}$;

- It will demonstrate the effectiveness of new technologies for heating, control, diagnostics, cryogenics and remote maintenance, applied to fusion reactors;

- It will achieve a deuterium-tritium plasma capable of self-sustainment; 
- It will test the breeding blanket technology and so the possibility of producing the tritium needed for the fusion reactions inside the reactor itself;

- It will demonstrate the safety characteristics of a fusion device.

DEMO will largely build on the ITER experience; indeed, its construction will start after several years of ITER operation. Now, the design of DEMO is based on five main objectives [3]:

- Conversion of fusion thermal power into electricity for several hundreds of megawatts;

- Achievement of tritium self-sufficiency (the tritium produced through the breeding blanket technology is higher than that consumed during the fusion reaction);

- Reasonable availability of up to several full-power years;

- Minimization of radioactive wastes, with no-long-term storage;

- Extrapolation to a commercial fusion power plant.

Considering the achievements that both the facilities should reach, the actual characteristic that distinguishes DEMO from ITER is the size and consequently the possibility of achieving higher values of fusion gain factor and longer fusion time. The fusion gain factor is defined as the thermal power produced inside the reactor during the fusion reaction divided by the thermal power delivered to the plasma during the operation. In particular, DEMO being bigger than its predecessor, it is designed to reach a fusion gain factor between 10 and 50 [1], while this value for ITER is foreseen to be around 10 [1]. One of the aims of current studies on this topic is to understand if this gain factor is high enough to allow a feasible supply of electrical energy to the grid, also because the electrical power systems of tokamaks like ITER or DEMO are larger and more complex than those of nuclear-fission power plants.

As for every tokamak, the main limitation for DEMO is the impossibility of maintaining the fusion reactions for an indefinite time [4]. This is an intrinsic characteristic of tokamaks, and it is related to the need of charging and discharging the central solenoid (CS) system that generates and confines the plasma current. This essentially means that the operation of DEMO is variable, and in particular, it is divided in several phases that will be presented in following section.

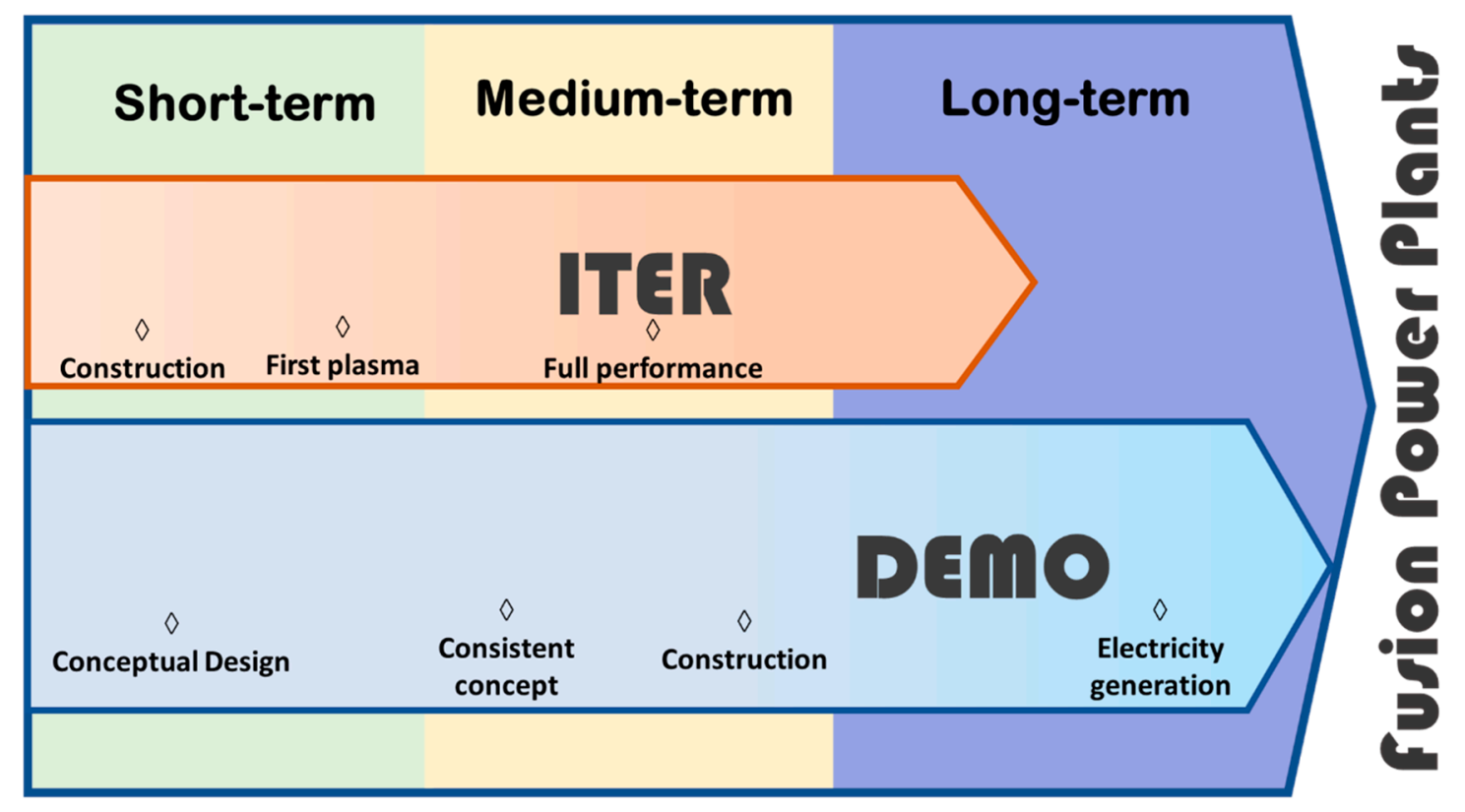

Figure 1. Summary of the EUROfusion Roadmap to fusion energy.

Now, the researchers are trying to understand how and how much the non-generation time can be reduced, even if the generation time is already foreseen to be at least one order of magnitude greater than the non-generation time. From the structural point of view, the need to optimize this 
characteristic led to the identification of two possible alternative configurations. The first one involves the direct coupling between the Primary Heat Transfer System (PHTS, the system that extracts the thermal power from the reactor walls) and the Power Conversion System (PCS), which reflects the variability of the thermal output into the electrical power output. The second one involves the indirect coupling between the PHTS and the PCS, which allows to decouple the variable thermal output from the electrical power output, with the interposition of an Intermediate Heat Transfer System (IHTS) with an Energy Storage System (ESS) based on molten salts [3], allowing a constant electrical power output at the generator level.

The article includes five sections. Section 2 defines the main characteristics of DEMO, and it introduces its operational phases. Section 3 focuses on the electrical generator, defining the limitations of the possible coupling configurations, through the analysis of the European Network of Transmission System Operators (ENTSO-E) requirements for generators. Section 4 presents three possible connection solutions for the DEMO facility, considering both the demand and the generation, providing an overview of the advantages and disadvantages of each solution. In Section 5, starting from the input and output power profiles, some features of the point of connection to the grid are evaluated. Section 6 resumes the conclusions of the study.

\section{DEMO Features and Operational Phases}

DEMO is foreseen to generate a fusion thermal power inside its reactor that has been evaluated to be in the order of 2 GWth [5]. Now, two solutions are under study for the thermal power extraction and accordingly for the PHTS. The first solution exploits a mature technology, which is the water cooling, also used in fission nuclear plants for its simplicity and reliability. The second solution instead foresees the use of helium for the PHTS, which seems to be promising for future applications but is still a relatively new technology. Conventionally, the first solution is identified as Water Cooled Lithium Lead (WCLL), where lithium lead refers to the technology adopted inside the reactor wall, and the second one as Helium Cooled Pebble Bed (HCPB) [6].

In case of direct coupling between the PHTS and the PCS, both the WCLL and the HCPB configurations provide for the implementation of a Rankine cycle for the thermal power conversion. This means that in case of HCPB, the helium cools the reactor (PHTS), and then, it exchanges the extracted power with the cycle working fluid, namely water. Moreover, in case of indirect coupling, the working cycle is a Rankine cycle, but in this case, the decoupling between the PCS and the PHTS makes the overall operation independent of the type of fluid circulating in the PHTS. From now on, more efforts are being focused on the WCLL configuration and so more data are available. This study will mostly refer to this solution. In any case, several results and procedures can be applied both to the WCLL and to the HCPB configurations.

For the limits of the tokamak technology, the thermal power is generated only during a portion of the operation time, which is generally identified as the "Burn Flat-Top" phase. A smaller power can be generated in the other phases for thermal inertia of the materials, nuclear reactions and other phenomena but always, as a consequence of the Burn Flat-Top. In the present configuration, this phase should last around $7200 \mathrm{~s} \mathrm{(2} \mathrm{h).} \mathrm{Between} \mathrm{each} \mathrm{burn} \mathrm{phase,} \mathrm{other} \mathrm{phases} \mathrm{are} \mathrm{conventionally} \mathrm{identified}$ during which no relevant thermal power is extracted. These phases are resumed in Table 1, with their respective duration.

For completeness, a brief description of the phases is reported. During CS pre-magnetization, the CS, that is, the core of the magnet system, is energized in order to be able to generate a power pulse strong enough to generate the plasma ignition that is represented by the Breakdown phase. Once the plasma has been generated inside the reactor, it must be heated up to the temperatures needed to have a sustainable rate of fusion reactions, and this is done during the Plasma Ramp-Up and Heating Flat-Top phases, mainly using the additional heating (AH) systems. Now, the most likely solution allows for the use of three technologies for the AH: Electron Cyclotron Resonance Heating (ECRH), Ion Cyclotron Resonance Heating (ICRH) and Neutral Beam Injection (NBI) [7]. When the temperature 
reaches the order of $10^{7} \mathrm{~K}$, the conditions inside the reactor allow to have self-sustained fusion reactions happening, and this identifies the Burn Flat-Top phase. During this phase, the power demand from the magnet system and the $\mathrm{AH}$ is minimum, while the thermal power generated is maximum. At the end of the burn phase, the reactor has to be brought back to the initial conditions avoiding shocks in the plasma, and this is done during the Plasma Ramp-Down and the Dwell time.

Table 1. Plasma phases.

\begin{tabular}{cccc}
\hline Phase Initial Time & Phase Final Time & Phase Duration & Phase Name \\
\hline$-500 \mathrm{~s}$ & $0 \mathrm{~s}$ & $500 \mathrm{~s}$ & CS pre-magnetization \\
$0 \mathrm{~s}$ & $1.4 \mathrm{~s}$ & $1.4 \mathrm{~s}$ & Plasma Breakdown \\
$1.4 \mathrm{~s}$ & $184 \mathrm{~s}$ & $\approx 183 \mathrm{~s}$ & Plasma Ramp-Up \\
$184 \mathrm{~s}$ & $194 \mathrm{~s}$ & $10 \mathrm{~s}$ & Heating Flat-Top \\
$194 \mathrm{~s}$ & $7394 \mathrm{~s}$ & $7200 \mathrm{~s}$ & Burn Flat-Top \\
$7394 \mathrm{~s}$ & $7540 \mathrm{~s}$ & $\approx 146 \mathrm{~s}$ & Plasma Ramp-Down \\
$7540 \mathrm{~s}$ & $7740 \mathrm{~s}$ & $200 \mathrm{~s}$ & Dwell time \\
\hline
\end{tabular}

The whole operation of DEMO, in terms of input and output power of the facility, can be described referring to the phases resumed in Table 1.

For what concerns the power needed to operate the facility, two types of loads have to be considered: the steady-state loads and the pulsed loads. The first ones require a steady-state $50 \mathrm{~Hz}$ voltage input, i.e., auxiliaries, cryogenics, pumps or compressors for the PHTS, etc. The pulsed loads, i.e., magnet system and AH devices, have to be supplied with variable voltage waveforms, and so, they will be provided with complex power conversion systems that are currently under study. The steady state and pulsed loads are supplied by dedicated substations and distribution systems [5], respectively, the Steady State Electrical Power System (SS EPS) and the Pulsed Power Electrical Power System (PP EPS). By convention, the PP EPS is identified with its two subsystems, the Coil Power Supply Pulsed EPS (CPSP EPS) and the Heating Power Supply Pulsed EPS (HPSP EPS), which supply the magnets system and the AH devices, respectively. From the generation point of view, instead, the generator subsystem is here conventionally referred to as Electrical Generator EPS (EG EPS). The connection node and the High Voltage/Medium Voltage (HV/MV) transformation sub-station are defined as High Voltage Switchyard EPS (HVS EPS).

In order to have a preliminary overview of the facility's demand, Figure 2 presents a qualitative profile of the input active power, derived from the data available in the EUROfusion private database.

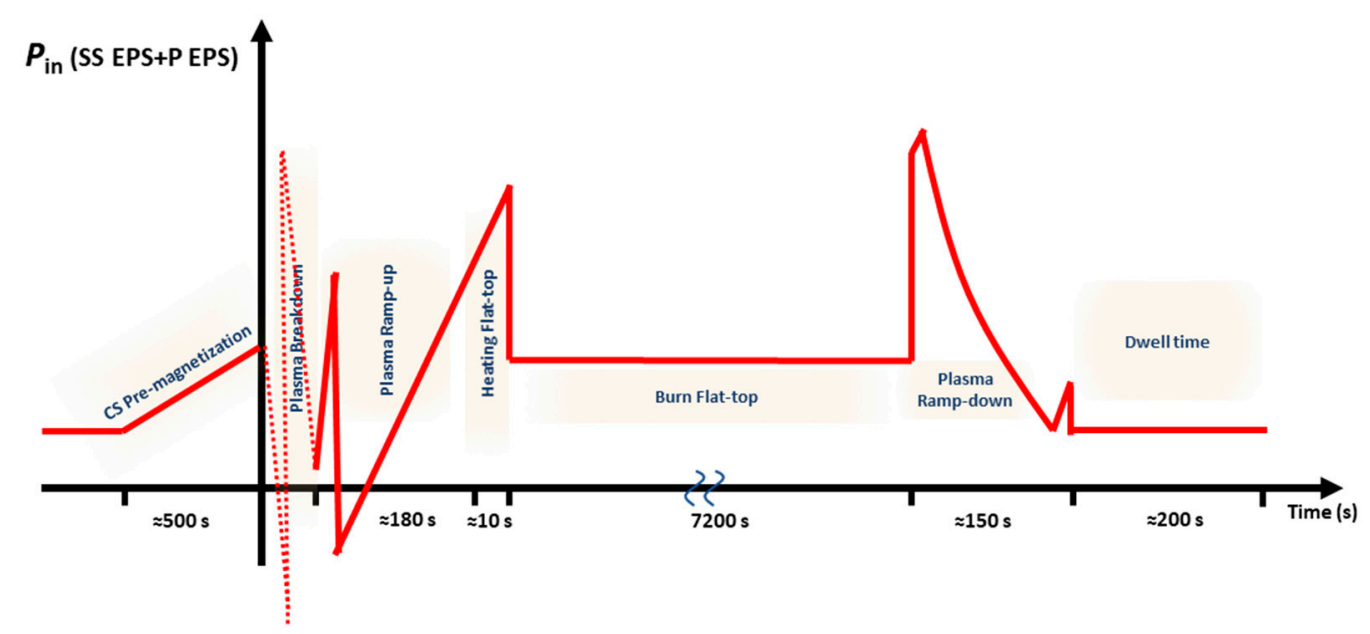

Figure 2. Qualitative profile of DEMOnstration power plant (DEMO) active power demand during one cycle. 
Regarding the power generation, of course, the direct and indirect coupling cases have to be separately considered to evaluate the output power profiles.

Despite being the simplest solution form the constructive point of view, the direct coupling between the PHTS and the PCS leads to several thermomechanical and electrical concerns. Regarding the thermomechanical aspects, several studies, not public but available for the authors as researchers involved in DEMO project on the Eurofusion database, assess the impossibility of operating the turbine with a completely direct coupling with the reactor. Firstly, the thermomechanical stresses due to the abrupt changes in the steam mass flow rate would be unsustainable for the turbine, leading to premature failures of the turbine itself. To limit these cyclical stresses, the maximum steam mass flow rate has to be reached with a ramp. In particular, the nominal power of the turbine has to be reached with an increase of $10 \%$ of the nominal power per minute. Another limit concerns the minimum power at which the turbine can be operated, that is, the minimum steam flow rate that can be supplied. In this case, the problem affects both the thermomechanical and the electrical aspects. Indeed, from the thermomechanical point of view, the turbine would suffer from the cyclical start and stop procedures, while from the electrical point of view the generator cannot lose the synchronism with the grid, so it has to be kept spinning. This means that the turbine has to be supplied with the proper mass flow rate of steam also during the non-generation time. In particular, the minimum power of the turbine has been set to $10 \%$ of the nominal power. During the reactor non-generation time, the solution currently under study allows for the implementation of a small electrically heated molten-salts loop, designed to provide the proper mass flow rate of steam to the turbine.

Referring to the latest studies, not public but available for the authors as researchers involved in DEMO project on the Eurofusion database, the nominal power of the steam turbine has been evaluated to be around $790 \mathrm{MW}$. Considering a 5\% value for the losses due to the coupling between the turbine and the synchronous generator, the nominal value for the electrical power output can be estimated to be about $750 \mathrm{MW}$. Considering this nominal value and the limits previously reported in case of direct coupling between the PHTS and the PCS, the resulting active power profile is shown in Figure 3.

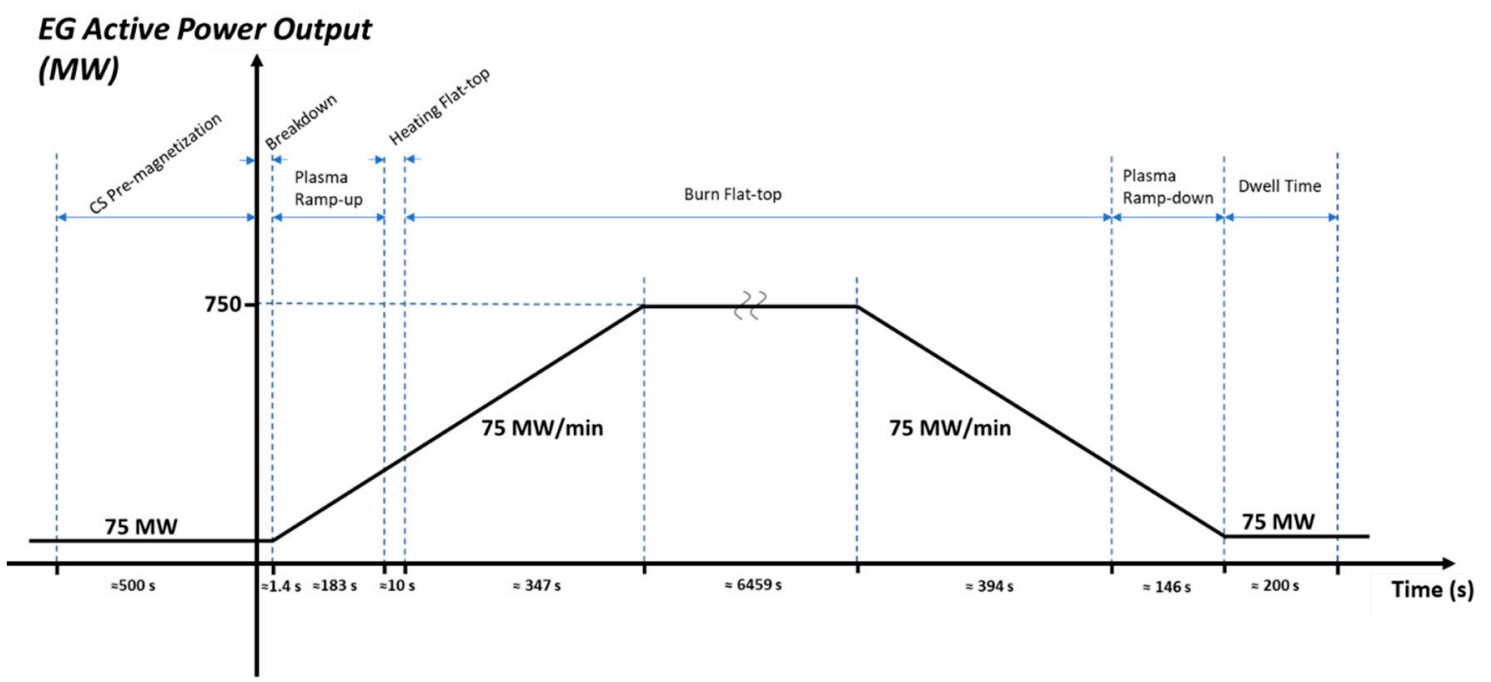

Figure 3. Electrical Generator (EG) active power output profile in case of direct coupling between the Primary Heat Transfer System (PHTS) and the Power Conversion System (PCS).

For the indirect coupling configuration, the situation is more complex from the constructive point of view, due to the IHTS and the molten-salts ESS, but the management of the turbine and of the generator is simpler. Indeed, in this case the steam flow rate supplied to the turbine can be maintained practically constant during the whole operation of the facility, like in a conventional power plant, minimizing both the thermomechanical and the electrical stresses. 
Referring to the latest studies available on the EUROfusion private database, the nominal power of the turbine in case of indirect coupling configuration is around $675 \mathrm{MW}$. Considering the same value used in the previous case for the efficiency of the turbine-synchronous generator coupling (0.95), the resulting active power output at the EG level is around $640 \mathrm{MW}$. The resulting profile is reported in Figure 4.

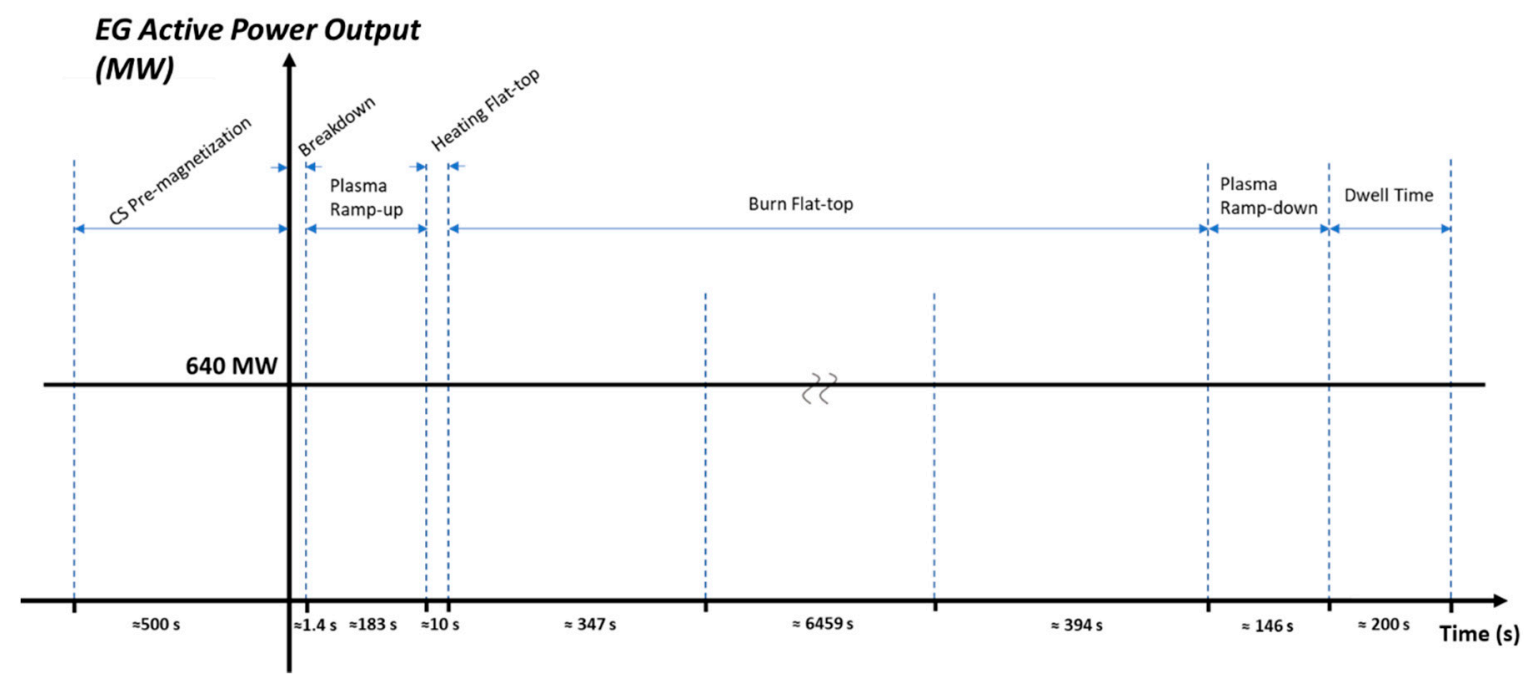

Figure 4. EG active power output profile in case of indirect coupling between the PHTS and the PCS.

\section{Connection of DEMO EG to the European Electrical Grid}

Being the first fusion reactor able to deliver electrical power to the external grid, DEMO has to face new issues with respect to its predecessors. Of course, as it has been mentioned before, DEMO is not a conventional power plant. However, since it uses a conventional thermodynamic cycle for the conversion and is connected to the grid through a synchronous generator, now it has to be considered as a conventional power plant from the regulatory point of view.

In reference to the current European power systems regulation, as a synchronous generator connected to the transmission grid, for a proper operation, DEMO has to fulfil the requirements set by the ENTSO-E. Specifically, these requirements are defined in the ENTSO-E Regulation of 14 April 2016 "Establishing a network code on requirements for grid connection of generators" (commonly said RfG network code) [8].

In what concerns synchronous generators, which in the code are referred to as synchronous Power Generating Modules (PGMs), the RfG distinguishes four categories, according to the voltage level of the connection and to power rating of the facility (identified as maximum capacity inside the regulation). The categories reported in the RfG are the following:

- Type A PGM, characterized by a voltage level at the connection point below $110 \mathrm{kV}$ and maximum capacity of $0.8 \mathrm{~kW}$ or more;

- Type B PGM, characterized by voltage level at the connection point below $110 \mathrm{kV}$ and maximum capacity between $1 \mathrm{MW}$ and $50 \mathrm{MW}$;

- Type C PGM, characterized by voltage level at the connection point below $110 \mathrm{kV}$ and maximum capacity between $50 \mathrm{MW}$ and $75 \mathrm{MW}$;

- $\quad$ Type D PGM, characterized by any voltage level at the connection point and maximum capacity higher than $75 \mathrm{MW}$.

Therefore, for the specific case of DEMO, the requirements for Type D synchronous PGMs, namely those with a power rating higher than $75 \mathrm{MW}$, have to be considered. The requirements for Type D PGMs deal with: 
1. Frequency stability;

2. Limited frequency sensitive mode-over-frequency;

3. Admissible power reduction;

4. Limited frequency sensitive mode-under-frequency;

5. Frequency sensitive mode;

6. Capability of maintaining constant output;

7. System restoration;

8. Voltage stability;

9. Robustness;

10. General system management;

11. Specific requirements for synchronous PGMs.

Since the research is still in a preliminary design phase, there is not enough information to analyze all the aspects of the RfG. Therefore, for the purpose of this study, we will refer only to the main aspects, i.e., those that can have an actual impact on the current design choices. In particular, we will consider the items in the list above from 1 to 7 and 11. The items from 1 to 6 deal with power generation and control, while items 7 and 11, respectively, deal with the capability of restoring the system after a shutdown of the grid and with the reactive power capability of the synchronous generator.

\subsection{Constant Power Output Requirement}

The frequency stability requirement defines the frequency ranges, and respective time intervals, for which the PGM shall be able to remain connected and operate in the grid. Moreover, for item 6 in the list above, in this range, the PGM shall be capable of maintaining a constant output at its target active power value. While in case of indirect coupling this requirement is fulfilled (Figure 4), it represents the strongest practical limitation to the choice of a direct coupling configuration, due to the variability of the EG power output. Referring to the profile of the active power output reported in Figure 3, considering the large variation of the output power (from 75 to $750 \mathrm{MW}$ ) and the fast variation in time, no compensation could be possible on the electrical side. Therefore, if the direct coupling configuration has to be adopted, the only way to fulfill the constant output requirement is to act on the mechanical power and so on the thermal power provided to the turbine or to act outside the main turbine-generator (TG) group. Therefore, the constant output power can be achieved in two main ways: One way is to make the TG work at the same active power set-point during all the phases; the other way is to couple the TG with an auxiliary generation set, even based on a totally different technology.

The first way can be seen as a category of solutions, all based on feeding a constant mass flow rate of steam to the steam turbine. Feeding always the same mass flow rate to the steam turbine essentially means to have an auxiliary steam generator. Actually, in the direct coupling PHTS-PCS scheme, one auxiliary steam generator is already foreseen, but it only provides the steam needed to operate the TG at the $10 \%$ of its nominal power (as mentioned in the previous section). Coherently with the volumes involved, the ESS loop could be foreseen with a higher rating, in order to guarantee up to $100 \%$ of the nominal mass flow rate of steam to the TG. Since the ESS loop is currently foreseen to be fed by an electric heater, it has to be considered that the net output power of the facility will be lower. Moreover, the convenience of the direct coupling approach, which is mainly the simplicity of construction with respect to the indirect coupling one, could be no longer that evident. Nonetheless, this solution could be even more complex than the pure indirect cycle.

The second solution is even more complex and expensive with respect to the first one, since it requires the implementation of a secondary generation set able to compensate the generation during the non-production time of the reactor. Since the power rating of the auxiliary generation set should be comparable with that of the first one, this kind of solution appears to be unlikely to be implemented. At the connection with the external grid, the sum of the two generation sets would appear as a good 
approximation of a conventional Type D PGM, but actually, it would have an installed maximum power capacity which is around two times the nominal one.

\subsection{Power Control Capability Requirements}

The Limited Frequency Sensitive Mode-Over/Under frequency (LFSM-O/U) is an operation policy, activated by the Transmission System Operator (TSO) when the grid is an emergency state of over/under frequency and needs a fast decrease/increase of active power generation [9]. The Frequency Sensitive Mode (FSM) instead represents the ordinary operating mode of a PGM, in which the power output changes in response to a change in the frequency of the system, in such a way that it supports the recovery of the target frequency [10]. Therefore, all the points from 2 to 5 deal with the power control capability of the PGM. In particular, the most restrictive value defined in the RfG is $10 \%$ (so $\Delta \mathrm{P}_{\max }=0.1 \mathrm{P}_{\max }$ ) power control capability, which is provided for the FSM operating mode. It is important to stress out that the provision of the power control capability is limited by the minimum regulating level and by the maximum capacity of the PGM. In addition, the ambient conditions and the limitations on the operation near maximum capacity at low frequency of the PGM have to be considered.

Regarding the indirect cycle configuration, while the stable and continuous operation with a fixed active power set point should be guaranteed thanks to the IHTS, evaluations on the molten salts ESS-water coupling should be performed, in order to define the potential of the system in terms of rate of change of active power.

In case of direct coupling, moving from the profile in Figure 3, the continuous and dashed red lines in Figure 5 represent the profile that the generation should be able to maintain in order to operate in the European Network as a conventional Type D synchronous PGM. In particular, the continuous line represents the maximum capacity operation that by definition the PGM should be capable of providing continuously during the ordinary operation in the grid. The dashed line instead defines the active power lower limit for the operation in case of FSM, with the power variation set to the maximum value of $10 \%$ of the maximum capacity as a conservative solution, since its actual value depends on the agreement carried out between the facility owner and the relevant TSO.

Active Power Output (MW)

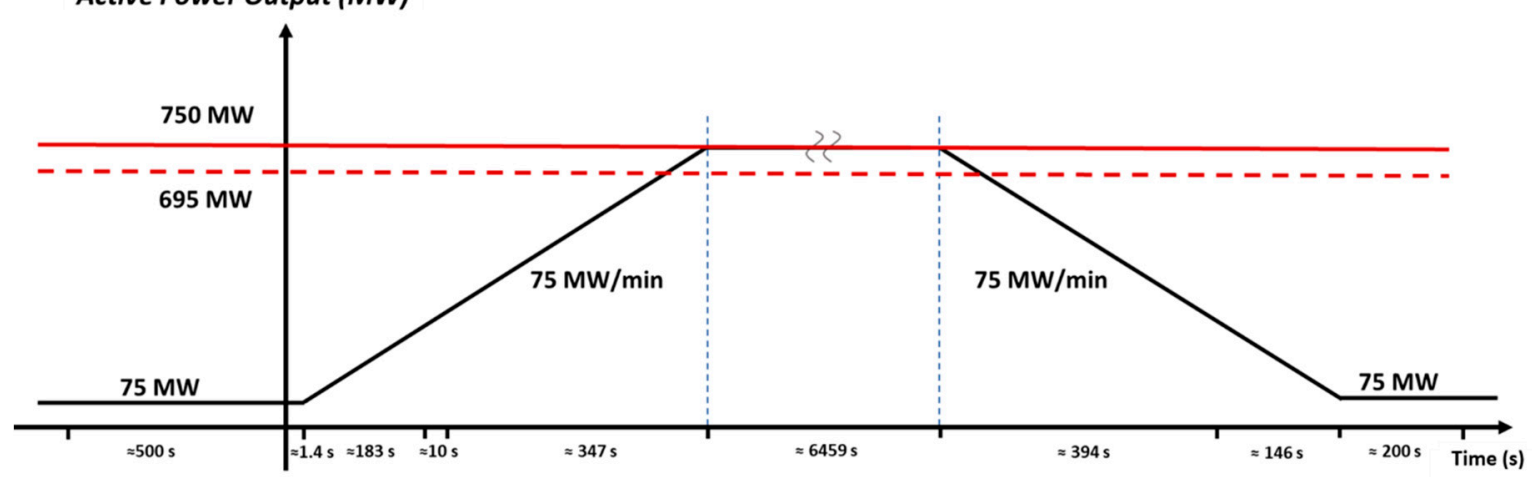

Figure 5. Active power output adapted to the European Network of Transmission System Operators (ENTSO-E) requirements.

Concerning the red dashed line, the capability of lowering the active power output, since the fusion thermal power has to be extracted in any case from the reactor (referring to generation time), it is sufficient to use suitable bypass valves upstream from the steam turbine. Of course, the solution must be compliant with the capacity of the condenser downstream the turbine that should be able to elaborate additional flows of steam and not only the double-phase flow coming from the turbine itself. For the direct coupling configuration, this condition should be already fulfilled since during the power ramps a variable portion of steam bypasses the steam turbine, so there could be no need for adjustments in these terms. 


\subsection{System Restoration Requirements}

Concerning the system restoration, the relevant TSO may require the black start capability. A PGM with the black start capability shall be able to restart from a shutdown without the external grid electrical energy supply. This kind of service may be possible in case of indirect coupling, depending on the size of the reserve and on the duration of the shutdown, but it is not compliant with the operation in case of direct coupling. Indeed, in case of a network shutdown, the reactor is likely to be shut down in turn, since its operation and security strongly depend on the network supply. Moreover, for the same reason, the reactor cannot be restarted without the external grid, leading to the impossibility of a black start of the PGM.

\subsection{Specific Requirements for Type D Synchronous PGMs}

The operation of a Type D synchronous PGM requires additional specifications, mainly in terms of voltage stability and so about reactive power capability at and below the maximum capacity. From these requirements, we can extrapolate the maximum value for the $\mathrm{Q} / \mathrm{P}_{\max }$ ratio that a Type $\mathrm{D}$ synchronous PGM connected to the European electrical grid should be able to provide. This means that we can evaluate a preliminary power rating, in terms of apparent power, of the synchronous machine that has to be coupled with the steam turbine. Since the maximum value set in the RfG for this ratio is 0.65 , which means $\cos \phi=0.84$ (a common value for machines of this rating that can be found also in literature [11]), we will have that:

- In case of direct coupling, the maximum active power is equal to $750 \mathrm{MW}$ during the burn phase; this means that the generator has to be able to supply $\mathrm{Q}_{\max \_ \text {direct }}=0.65 \cdot \mathrm{P}_{\max } \approx 490 \mathrm{MVAr}$, and this results in an apparent power rating of the machine of around $900 \mathrm{MVA}$;

- In case of indirect coupling, the maximum active power is equal to $640 \mathrm{MW}$; this means that the generator has to be able to supply $Q_{\max \_ \text {indirect }}=0.65 \cdot P_{\max } \approx 416 \mathrm{MVAr}$, and this results in an apparent power rating of the machine of around 765 MVA.

\section{Layout Options for HV Switchyard Including the Generator}

The results reported in this section take as reference the regulation on the connection of new facilities provided by the Italian TSO, Terna [12], considering that the Italian policies are based on the ENTSO-E prescriptions, so this approach is more conservative with respect to an approach based on the European requirements.

Relying on the guidelines provided by Terna, the connection of new facilities to the national grid must be planned in accordance with a proper procedure that is outlined in Figure 6.

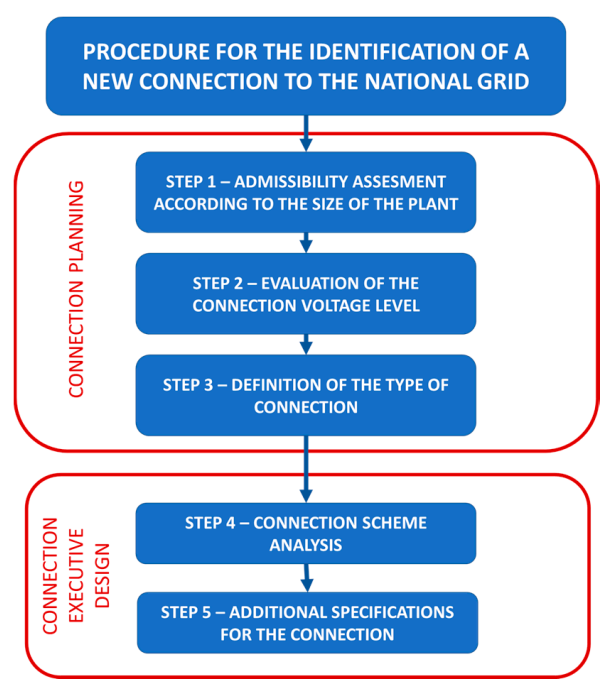

Figure 6. Procedure for the connection of generators and consumers to the European grid. 
In particular, considering that the project is still in a preliminary design phase, the first three steps reported in Figure 6 will be considered in the analysis and so those that deal with the connection planning and not with the executive design, which are:

- The definition of the minimum value of power rating of the facility for which the connection has to be implemented in the transmission network, which is $10 \mathrm{MW}$; in particular, in case of both generation and demand facilities, the value to consider is the highest between the maximum injection and the maximum demand.

- The definition of the voltage level of the point of connection of the new facilities.

- The definition of the type of scheme to be implemented for the connection of the new facilities.

For the definition of the voltage level and of the connection scheme, the technical document [12] provides a table summarizing standard solutions, partially reported in Table 2.

Table 2. Standard solutions for voltage level and connection scheme of new generation or demand facilities.

\begin{tabular}{ccccc}
\hline \multirow{2}{*}{ Power Rating of the Facility } & Nominal Voltage Level & \multicolumn{2}{c}{ Standard Solutions } \\
\cline { 3 - 4 } & & & Radial & In-and-Out \\
\hline \multirow{3}{*}{ Generation } & $100-250 \mathrm{MW}$ & $120-150 \mathrm{kV}$ & Yes & No \\
& $200-350 \mathrm{MW}$ & $220-380 \mathrm{kV}$ & Yes & Single busbar + bypass \\
& $>350 \mathrm{MW}$ & $380 \mathrm{kV}$ & Yes & Double bus bar \\
\hline \multirow{2}{*}{ Demand } & $20-50 \mathrm{MW}$ & $120-150 \mathrm{kV}$ & Yes & Single busbar \\
& $30-100 \mathrm{MW}$ & $120-150 \mathrm{kV}$ & Yes & Single busbar \\
& $>100 \mathrm{MW}$ & $220-380 \mathrm{kV}$ & Yes & Single busbar + bypass \\
\hline
\end{tabular}

The in-and-out connection is performed with the implementation of a new station on an existing transmission line. This means that the new station is supplied by two different transmission lines, coming from two different nodes. Therefore, the facility can ideally operate even when one of the lines is out of service. The radial connection is similar to the in-and-out one, but the connection starts from an existing station of the transmission line. This solution is generally adopted when the distance between the existing station and the facility is lower than $10 \mathrm{~km}$.

Keeping in mind this simplified procedure to evaluate the connection characteristics, three possible configurations have been considered for the connection of DEMO to the European transmission grid:

- Single Point of Delivery (POD); EG EPS, SS EPS and PP EPS are connected to the same HV Switchyard;

- Double POD with EG-dedicated node; SS EPS and PP EPS are connected to one HV Switchyard, while the EG EPS is connected to another HV Switchyard;

- Double POD with PP EPS-dedicated node; SS EPS and EG EPS are connected to one HV Switchyard, while the PP EPS is connected to another HV Switchyard.

In the following subsections these solutions will be analyzed starting from power profiles elaborated in the DIgSILENT PowerFactory simulation environment [13], moving from the data available on the EUROfusion database in terms of production and consumption of the facility. Concerning the demand, the data refers to the assisted breakdown scenario, which allows for the use of EHCR during the breakdown phase to assist the magnets system, lowering the power peak required for the plasma ignition.

\subsection{Single $P O D$}

The single POD configuration is the one that requires the simplest implementation. In case of single POD, DEMO would fall in the category of power generating/demanding facilities. This means that the value of power to be considered in the evaluation of the connection scheme is the highest between the generation maximum power and the demand maximum power [12]. 
In Figure 7, the preliminary active power profiles are presented, in case of direct and indirect coupling.

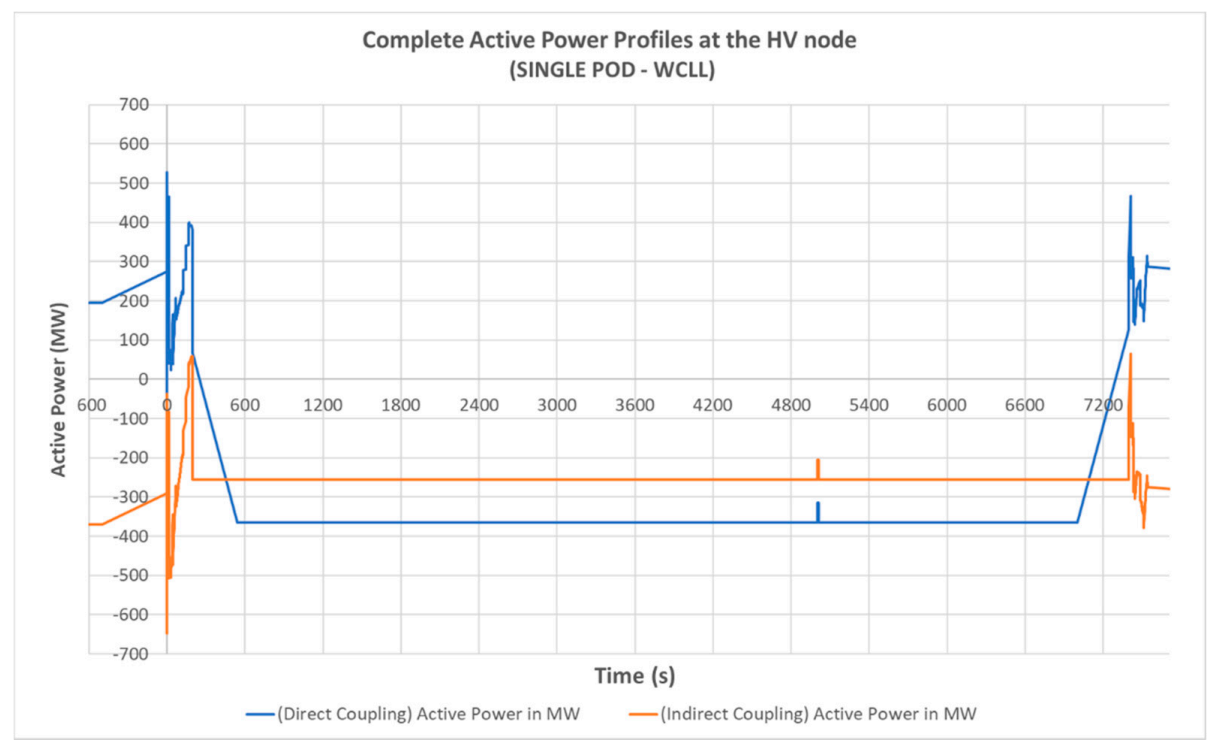

Figure 7. Power profiles at the HV node in case of single Point of Delivery (POD) configuration.

As shown in Figure 7, in case of direct coupling, the maximum power absorbed is around $500 \mathrm{MW}$, while the maximum power injected is around $360 \mathrm{MW}$. The maximum power is reached in "demand mode", and its value is higher than $100 \mathrm{MW}$. This means (considering Table 2) that the connection shall be performed at the highest available voltage level with the implementation of an in-and-out single bus-bar scheme with bypass. In case of indirect coupling instead, we can see that there is a peak of injected power of around $650 \mathrm{MW}$, which is higher than the peak of absorbed power, so the connection has to be performed at the highest available voltage level with the implementation of an in-and-out double bus-bar scheme (Figure 8). Of course, also the radial configuration can be adopted if allowed by the distance of the station.

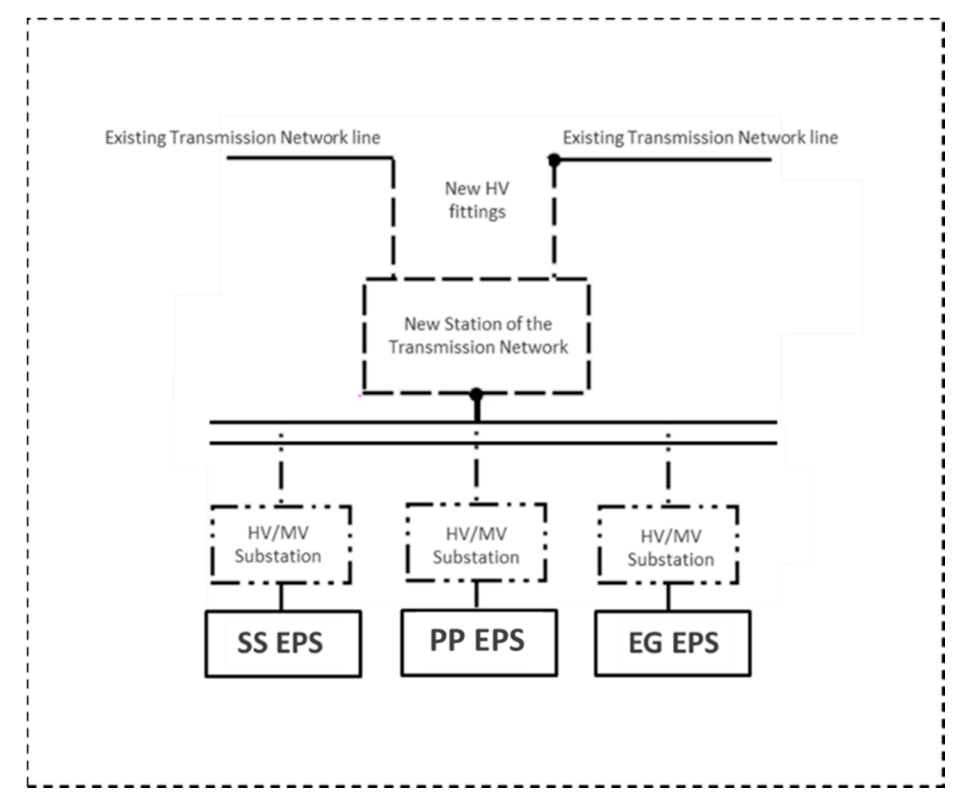

Figure 8. In-and-out double bus-bar connection scheme in case of single POD. 
With the implementation of a single POD, the node cannot be seen from the external grid point of view as a proper generation node. This means that the current legislation on the connection of generators (RfG code) to the grid cannot be applied. Moreover, this configuration is as easy to implement as it is dangerous, both for the operation of the facility and of the network. In fact, the high-power spikes absorbed by the converters to feed the PP EPS loads would jeopardize the functioning of the generator, since it would be the nearest source of power. This implies high transient electromechanical torque applied to the shaft of the TG, whose integrity could be seriously compromised, both for the magnitude and for the cyclicality of the resistive torque. To avoid this situation, an electrical ESS could be implemented upstream the PP EPS, to limit the power derivatives.

\subsection{Double POD with EG-Dedicated Node}

It is clear that the generator should be decoupled as much as possible from the PP EPS supply. One way to do so is by implementing a double POD solution, with one POD dedicated to the EG EPS and the second one for the SS EPS and PP EPS. Figure 9 shows the active power profile at the POD for the SS EPS plus PP EPS, and Figure 10 shows the generation profile of the EG EPS.

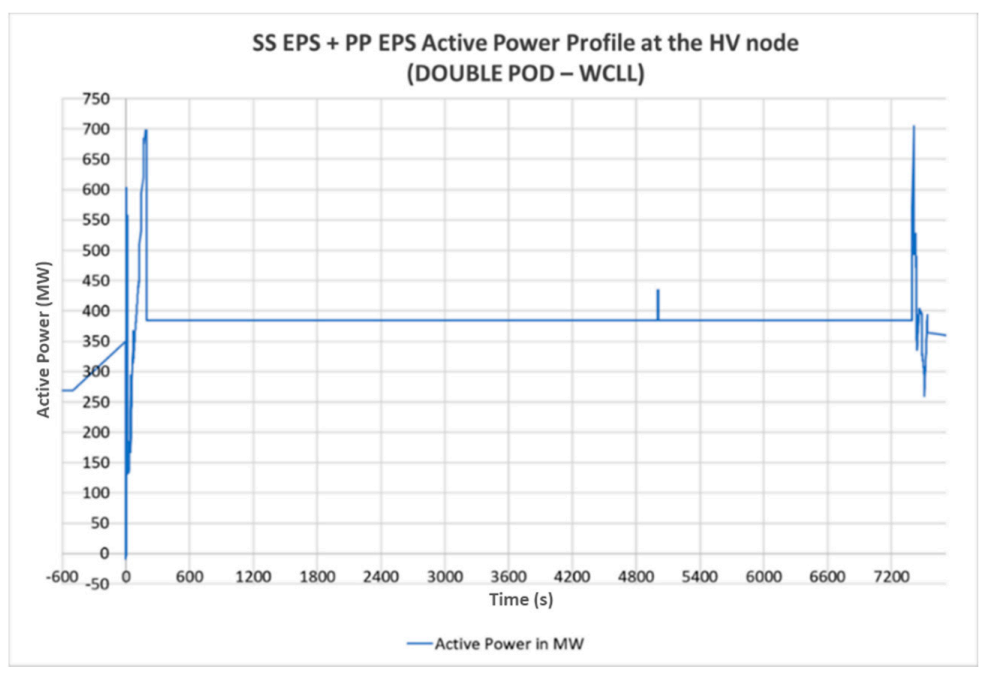

Figure 9. Power profile at the Steady State Electrical Power System (SS EPS) + Electrical Power System (PP EPS) HV node in case of double POD configuration.

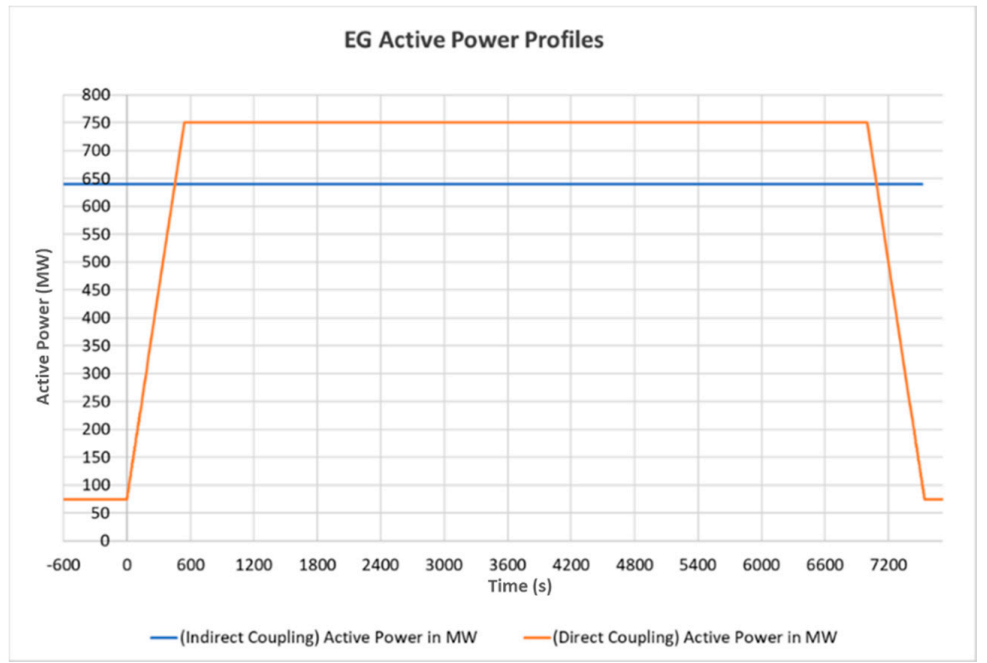

Figure 10. Power output of the generator in case of direct and indirect coupling. 
For convention, the power supplied by the external grid is represented as positive, while the power injected into the grid is negative. However, in Figure 10 the generator output power profile is defined from the generator point of view, so it is represented as positive. Of course, from the external grid point of view, that power is negative, since it is injected into the grid itself.

Regarding the SS EPS plus PP EPS POD, since the maximum power required from the grid is around $700 \mathrm{MW}$, the connection shall be performed at the highest voltage level available, with the implementation of an in-and-out single bus-bar scheme with bypass (see Table 2). Concerning the EG dedicated POD instead, the maximum power injected is $750 \mathrm{MW}$ in case of direct coupling and $640 \mathrm{MW}$ in case of indirect coupling. Therefore, independently from the coupling configuration, the connection shall be performed at the highest voltage level available, with the implementation of an in-and-out double bus-bar scheme.

Figure 11 reports the in-and-out connection schemes in case of double POD configuration with EG-dedicated node.

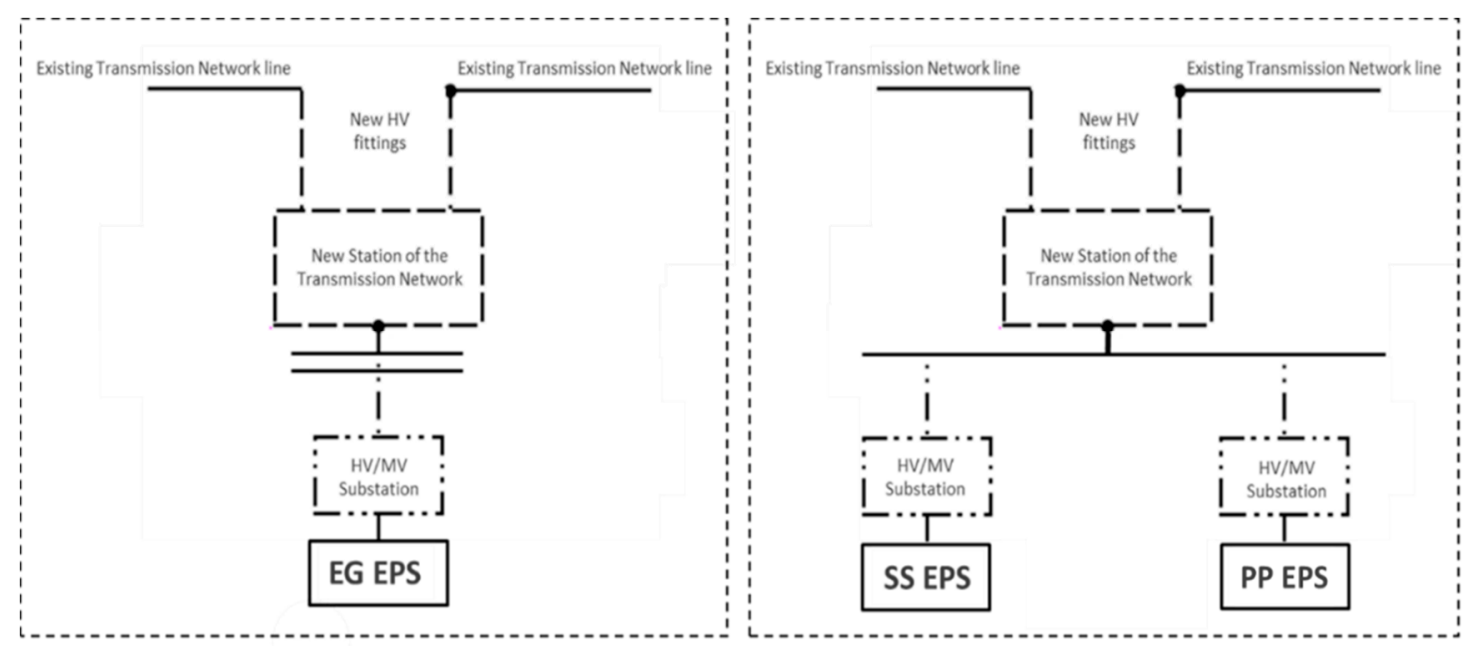

Figure 11. In-and-out connection scheme in case of double POD with EG-dedicated node.

As long as the generation node is well defined, all the requirements for the connection and operation of PGMs in the grid can be applied. Assuming the two POD to be fed by stations of the Transmission Network that are far enough from each other (in an electrical sense), in this configuration, the generator could operate more safely. Moreover, it could operate in line with the prescriptions for its specific category, bearing in mind the intrinsic limitations of the plant evaluated in Section 3.

Of course, from the demand point of view, the influence of the pulsed loads in this case is completely reflected to the transmission system.

\subsection{Double POD with PP EPS-Dedicated Node}

Another possible solution is the implementation of a double POD with one node dedicated to the PP EPS. Regarding the SS EPS plus EG EPS node, Figure 12 shows that, both in case of direct and indirect coupling, the maximum power is attained in generation mode, so the requirements to apply for the connection are those referred to the generation nodes. 


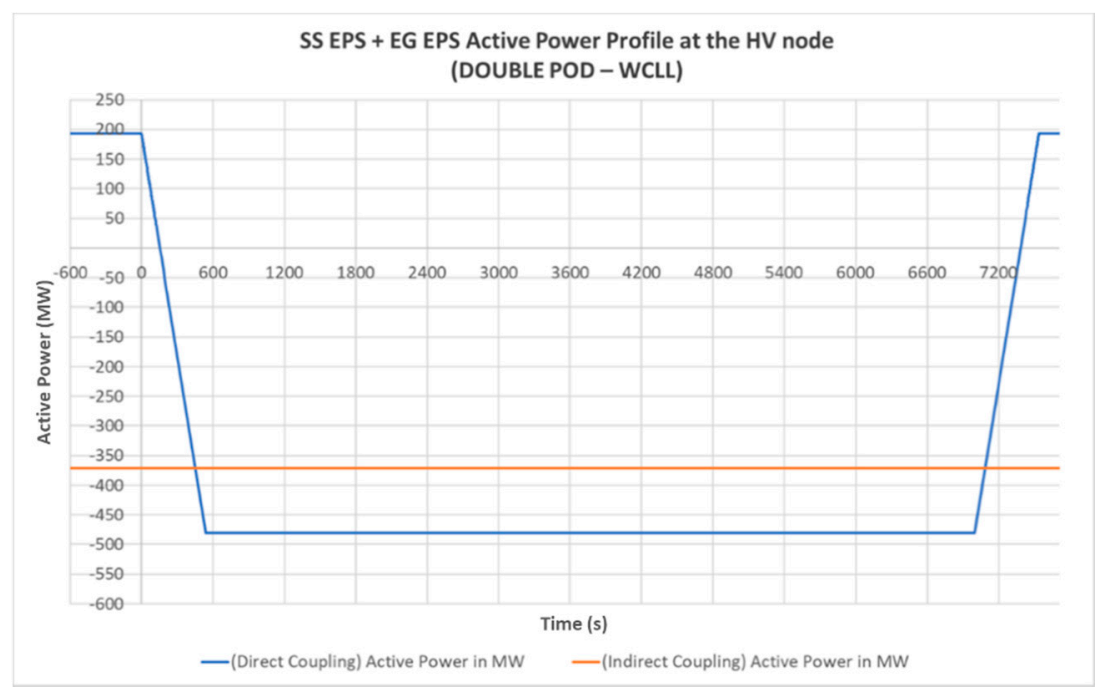

Figure 12. Power profiles at the SS EPS + EG EPS HV node in case of double POD configuration.

In particular, since the maximum power injected is higher than $350 \mathrm{MW}$, the connection shall be implemented at the highest available voltage level, with the in-and-out double bus-bar scheme (Table 2). In case of indirect coupling, the node appears to grid as a simple generation node, since the power needed for the SS EPS is entirely supplied by the EG EPS during all the operational phases. Therefore, of course the net output at the HV node level is lower than the previous configuration (EG-dedicated node), but the requirements for the connection and operation of PGMs in the grid are still applicable. In case of direct coupling instead, the node is both a generation and demand node since the EG EPS can only supply the whole power required from the SS EPS during the burn time.

Concerning the PP EPS-dedicated POD, the preliminary power profile at the HV node is presented in Figure 13.

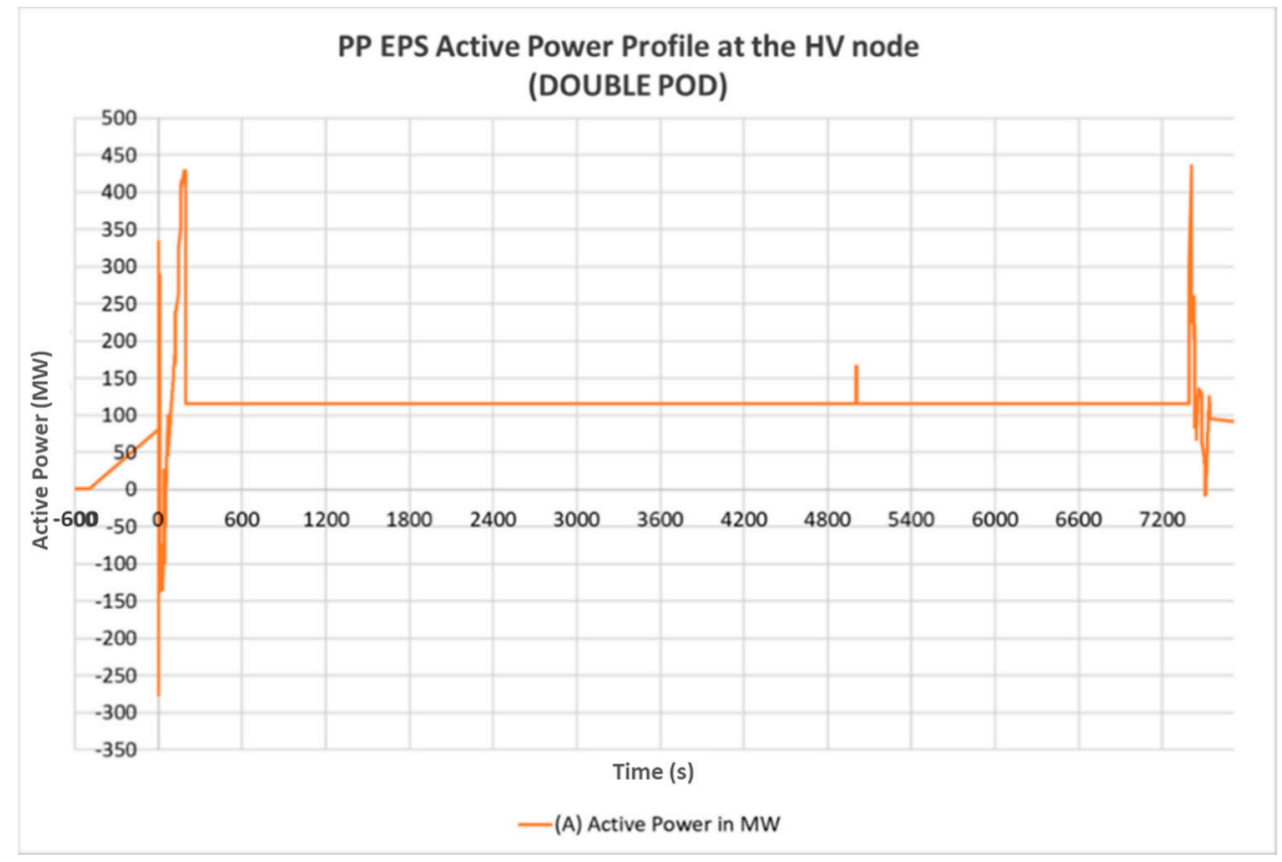

Figure 13. Power profile at PP EPS-dedicated HV node in case of double POD configuration. 
The maximum power absorbed at the node level is higher than $100 \mathrm{MW}$, so the connection shall be implemented at the highest voltage level available with the in-and-out single bus-bar scheme with bypass.

Figure 14 reports the in-and-out connection schemes in case of double POD configuration with PP EPS-dedicated node.

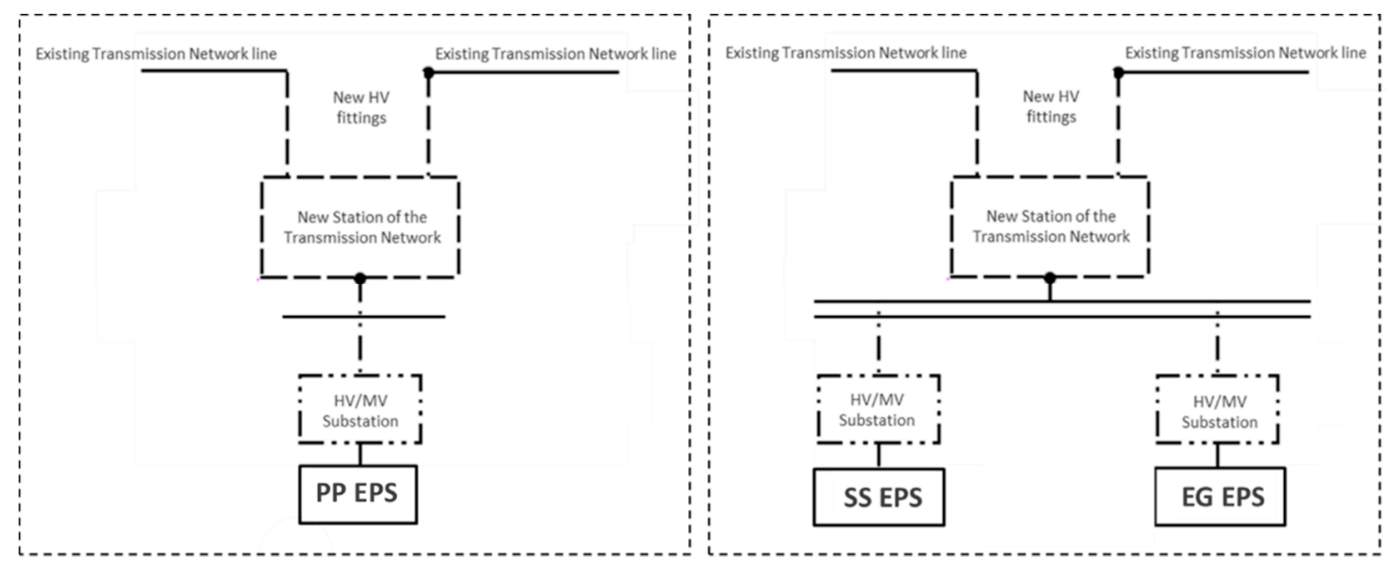

Figure 14. In-and-out connection scheme in case of double POD with PP EPS-dedicated node.

This configuration allows preserving the advantages of the previous case, decoupling both the SS EPS and the EG EPS from the supply of the PP EPS. Since the SS EPS requires practically constant input power, the generator can operate safely, supplying both the internal SS power system and the grid without further stresses and, in case of indirect coupling, as a conventional PGM connected to the grid. At the same time, the power peaks detected by the grid at the PP EPS POD, are of course lower than those related to the previous case where the SS EPS contribution makes them reach higher values.

\section{Features of the Point of Delivery (POD)}

This section reports further evaluations on the Point of Delivery (POD) that can be performed starting from the preliminary power profiles elaborated through DIgSILENT PowerFactory [13]. Indeed, talking about the active power profiles, some data can be extrapolated in order to have an idea of the main electrical features of the HV node (or nodes) to which DEMO should be connected. Concerning the reactive power instead, some general considerations are reported.

\subsection{Power-Frequency Control}

The requirements in terms of frequency control and admissible frequency variations are defined in the ENTO-E document "P1-Policy 1: Load-Frequency Control and Performance" [14]. The power-frequency characteristic of a power system is linked to its capability of limiting the frequency variation during an event of unbalance between generation and demand. The power-frequency variation is expressed in $\mathrm{MW} / \mathrm{Hz}$, so it physically represents the value of power variation in $\mathrm{MW}$ that causes a frequency variation of one Hz. Policy 1 [14] defines minimum and average power-frequency control characteristics in Continental Europe, which are respectively $15 \mathrm{GW} / \mathrm{Hz}$ and $19.5 \mathrm{GW} / \mathrm{Hz}$. Other important values of the Policy 1 [14] that we have to consider for the purpose of this analysis are the "minimum instantaneous frequency after a loss of generation" and the "maximum instantaneous frequency after a loss of load", which are respectively $49.2 \mathrm{~Hz}$ and $50.8 \mathrm{~Hz}$. This means that the maximum frequency deviation accepted in Continental Europe's synchronous area is $\pm 800 \mathrm{mHz}$ from the nominal value. However, it has to he stressed out that these values refer to the loss of a load or of a generation node; they do not refer to the ordinary operation of the grid. Indeed, to have a more reliable limit we should consider the maximum permissible quasi-steady-state frequency deviation that is set to $\pm 200 \mathrm{mHz}$. This frequency deviation also represents the limit for which all the available 
primary control reserves are expected to be fully activated. In fact, this value represents the maximum value that can be managed only relying on the primary frequency control.

Regarding our specific case, analyzing the active power profiles, it is possible to identify several power steps, mainly due to the functioning of the magnets system and of the AH devices. In particular, the most severe event in this sense is a $426 \mathrm{MW}$ active power step that occurs during the Plasma Ramp-up phase. The event, reported in Figure 15, is caused by the PP EPS operation.

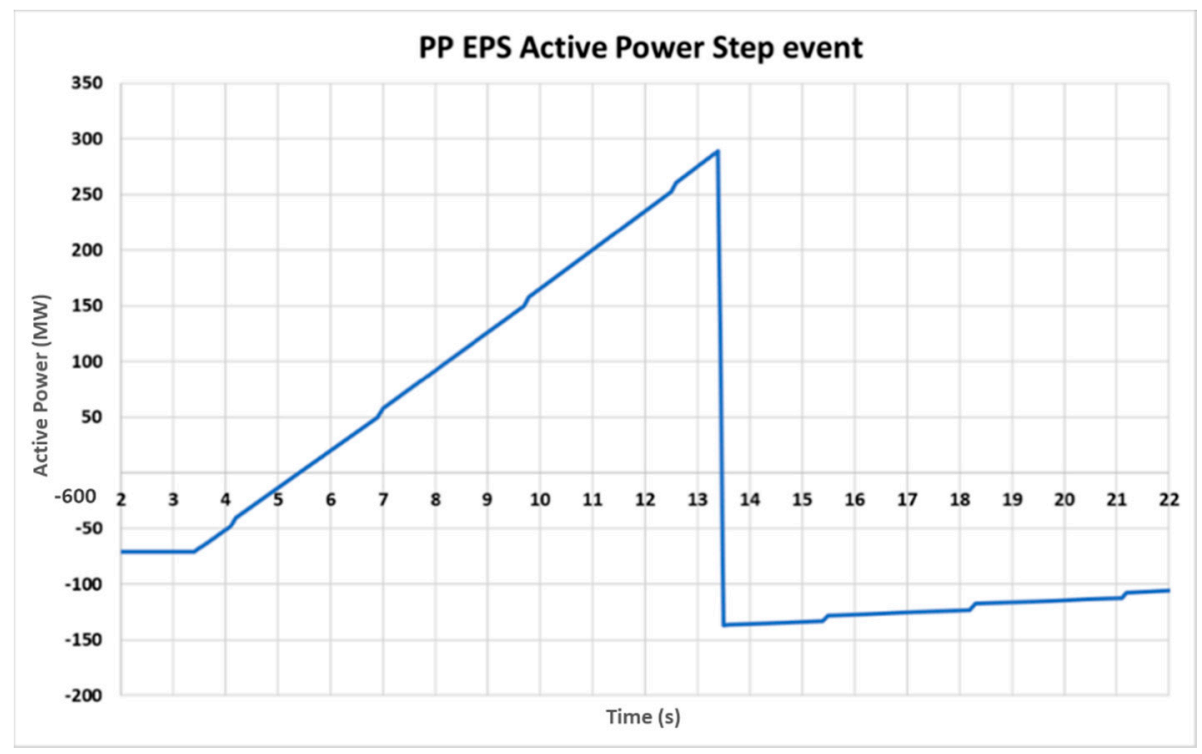

Figure 15. Active Power step required by the PP EPS during the Plasma Ramp-up phase.

Since this power step is caused by the PP EPS, it will be present in each configuration we considered in the previous section for the connection of DEMO to the electrical grid. Therefore, independently from the solution adopted, we have to evaluate the effect of this event on the grid. Considering the average value of power-frequency control characteristic for Continental Europe, we can calculate the frequency deviation caused by the step in Figure 15, which turns out to be around $+21.85 \mathrm{mHz}$, significantly lower than the $200 \mathrm{mHz}$ limit. Indeed, the $200 \mathrm{mHz}$ limit is reached in case of what is called Reference Incident inside the ENTSO-E documents, i.e., an unbalance between generation and demand of $3 \mathrm{GW}$. Nonetheless, this event refers to an unbalance condition distributed inside the European transmission grid and not to an unbalance in a single node. In this sense, the effect of a $426 \mathrm{MW}$ active power on the grid step must not be underestimated just because it theoretically does not generate a significant frequency deviation from the nominal value. Moreover, it must be stressed out that this step is not occasional but is cyclically repeated during DEMO operation, so this is another critical aspect.

\subsection{Voltage Drop and Short-Circuit Power}

Considering the reactive power, the connection node should be characterized through the short-circuit power. The link between the reactive power and the short-circuit power of the node is the voltage drop. It is well known that, for a fixed value of reactive power required by the facility, a higher short-circuit power of the connection node involves a lower voltage drop at node level. A first approximation of the voltage drop, expressed in per unit, $\Delta V$ [p.u.] caused by a variation of reactive power $\Delta \mathrm{Q}$ on a node with a short-circuit power $\mathrm{S}_{\mathrm{sc}}$ is provided by the well-known formula $\Delta \mathrm{V}$ [p.u.] $=\Delta \mathrm{Q} / \mathrm{S}_{\mathrm{sc}}$.

Since for now there is not any reliable data regarding the actual reactive power required for the operation of DEMO (mainly for what concerns the supply of the pulsed loads), we cannot really define a specific value for the short-circuit power needed at the connection node. Nevertheless, we can 
adopt an opposite approach starting from a reasonable value for the short-circuit power and from the requirements in terms of voltage drop defined by ENTSO-E. Bypassing the requirements in terms of power factor and so assuming DEMO to be a non-conventional demand facility, we can preliminarily estimate a reasonable maximum value of reactive power that the facility can demand from the grid.

Considering the studies carried out on ITER [15] and the size of DEMO with respect to its predecessor, the new facility is likely to be connected to a node with a short-circuit power rating in the order of at least 30-40 GVA. This value seems reasonable considering the configuration of the European electrical grid and also considering that nodes with this rating can be also found in Italy [16], where the shape of the country does not facilitate the creation of highly meshed grids (which means higher reliability and also higher short-circuit power).

Bearing in mind this range of values for the short-circuit power, we must define a range of values for the voltage drop that do make sense for the specific case. Considering that most of the reactive power will be required by the PP EPS (due to the power conversion systems), an estimation based on a maximum voltage drop at the connection node, around $\pm 3 \div 5 \%$, should be conservative and reliable. Indeed, the 3\% value for the voltage drop is the one assumed for the Pulsed Power Electrical Network (PPEN) in ITER, which corresponds to the PP EPS in our case, as mentioned in the System Requirements Documents of ITER (available in the ITER private database). Due to the considerably greater size of DEMO, it makes sense to assume a wider range for the voltage drop.

Defining the worst case in terms of short-circuit power and voltage drop, i.e., 30 MVA and 3\%, respectively, the resulting maximum reactive power demand from DEMO facility is around 900 MVAr.

Unlike the considerations reported for the active power related aspects, in this case the POD configuration affects the evaluations. As already mentioned, the main concerns in terms of reactive power absorption come from the power electronic devices needed to supply the magnets system and the AH and so the pulsed loads. Therefore, if the PP EPS is isolated from the other substations, which means we are adopting the third configuration (see Section 4.3), we can consider only its node as an unconventional demand node, and we can cut the contribution of the SS EPS to the total reactive power required from that specific node. If the first configuration is considered instead (see Section 4.1), since the EG EPS could not be operated as a conventional PGM in any case, the possibility of using it for the internal reactive power compensation is not to be excluded, still bearing in mind the drawbacks of this solution.

\section{Conclusions}

Considering the state of the art of the DEMO project, the present work has been carried out with the purpose of providing some cause for reflection on the choices that have been made and that will be made; it is not meant to define final design solutions. In fact, it is important to stress out that at this stage of the design, the data is continuously questioned, so the analyses carried out for the realization of this article aim at providing guidelines, procedures and addresses.

The data available from the studies that are being performed in research centers all over Europe allowed a first estimation of the input and output power profiles of the facility. Through the analysis of these profiles, it has been possible to evaluate the limitations of the facility, both in case of direct and indirect coupling between the PHTS and the PCS, from the generator operation point of view. In this sense, the conclusion is that the direct coupling configuration, despite being the simplest from the constructive point of view, would lead to the impossibility of considering DEMO as a conventional power plant, unless significant adjustments are foreseen. In any case, the eventuality that DEMO could be considered as a non-conventional generation and demand facility is not to be excluded. In this sense, no matter which configuration will be selected, the European grid could allow the operation of the facility. However, this would not be in line with the purpose of the whole roadmap, since it would lead to a single facility that is allowed to operate freely in the grid. If designed on these terms, DEMO would not be a model for real fusion power plant prototypes; it would be a model for a new generation of non-dispatchable generation plants. 
Regarding the connection configuration and characteristics, this article presented three possible solutions. In particular, the solution that allows for the implementation of an EG-dedicated point of delivery, even if it is theoretically not in line with the idea at the base of DEMO, which is the self-sustainment of its loads, is the one that shows more advantages. From the grid connection point of view, the generator could be operated in line with the requirements for its category, being the node a pure generation node. From the facility point of view instead, the turbine-generator group would be decoupled from the power spikes absorbed and injected by the PP EPS, guaranteeing a longer life of the mechanical components.

Author Contributions: Conceptualization, M.C.F. and M.P.C.; methodology, M.C.F. and M.P.C.; software, M.P.C.; validation, M.C.F., A.L. and S.C.; formal analysis, M.C.F. and M.P.C.; investigation M.P.C.; resources, A.L. and S.C. data curation, M.P.C. and A.L.; writing-original draft preparation, M.P.C.; writing-review and editing, M.P.C. and M.C.F.; visualization, M.P.C.; supervision, M.C.F., A.L. and S.C.; project administration, M.C.F., A.L. and S.C.; funding acquisition, M.C.F., A.L. and S.C. All authors have read and agreed to the published version of the manuscript.

Funding: This work has been carried out within the framework of the EUROfusion Consortium and has received funding from the Euratom Research and Training Programme 2014-2018 and 2019-2020 under grant agreement No 633053. The views and opinions expressed herein do not necessarily reflect those of the European Commission.

Conflicts of Interest: The authors declare no conflict of interest.

\section{References}

1. EUROfusion, European Research Roadmap to the Realization of Fusion Energy. 2018. Available online: https://www.euro-fusion.org/eurofusion/roadmap/ (accessed on 30 March 2020).

2. ITER. What Will ITER Do? Available online: https://www.iter.org/sci/Goals (accessed on 30 March 2020).

3. Barucca, L.; Ciattaglia, S.; Chantant, M.; Del Nevo, A.; Hering, W. Status of EU DEMO Heat Transport and Power Conversion Systems. Fusion Eng. Des. 2017, 136, 1557-1566. [CrossRef]

4. Morris, J.; Kovari, M. Time-dependent power requirements for pulsed fusion reactors in system codes. Fusion Eng. Des. 2017, 124, 1203-1206. [CrossRef]

5. Federici, G.; Bachmann, C.; Barucca, L.; Biel, W.; Boccaccini, L.; Brown, R.; Bustreo, C.; Ciattaglia, S.; Cismondi, F.; Coleman, M.; et al. DEMO design activity in Europe: Progress and updates. Fusion Eng. Des. 2018, 136, 729-741.

6. Ciattaglia, S.; Federici, G.; Barucca, L.; Lampasi, A.; Minucci, S.; Moscato, I. The European DEMO Fusion Reactor: Design Status and Challenges from Balance of Plant Point of View. In Proceedings of the 17 IEEE International Conference on Environment and Electrical Engineering (EEEIC 2017), Milan, Italy, 6-9 June 2017.

7. Lampasi, A.; Minucci, S. Survey of Electric Power Supplies Used in Nuclear Fusion Experiments. In Proceedings of the 17 IEEE International Conference on Environment and Electrical Engineering (EEEIC 2017), Milan, Italy, 6-9 June 2017.

8. ENTSO-E. COMMISSION REGULATION (EU) 2016/631 of 14 April 2016 Establishing a Network Code on Requirements for Grid Connection of Generators; ENTSO-E: Brussels, Belgium, 2016.

9. ENTSO-E. Limited Frequency Sensitive Mode, ENTSO-E Guidance Document for National Implementation for Network Codes on Grid Connection, 31 January 2018; ENTSO-E: Brussels, Belgium, 2018.

10. ENTSO-E. Frequency Sensitive Mode, ENTSO-E Guidance Document for National Implementation for Network Codes on Grid Connection, 7 November 2017; ENTSO-E: Brussels, Belgium, 2017.

11. Fitzgerald, A.; Kingsley, C.; Umans, S. Electric Machinery; McGraw-Hill Education; McGraw-Hill: York, NY, USA, 2002; Chapter 5.5; pp. 293-294.

12. Terna, Guida Agli Schemi di Connessione. Attachment A2 of Terna Network Code, July 2015; Terna: Rome, Italy, 2015. Available online: https://download.terna.it/terna/0000/0105/19.pdf (accessed on 30 March 2020).

13. DIgSILENT. Load Flow Analysis. Available online: https://www.digsilent.de/en/load-flow-analysis.html (accessed on 30 March 2020).

14. UCTE. Continental Europe Operation Handbook P1, Load-Frequency Control and Performance; UCTE: Brussels, Belgium, 2009. 
15. Benfatto, I. Power converters for ITER. Comput. Sci 2006, 231-247. Available online: http://cds.cern.ch/record/ 987554/files/p231.pdf?version=1 (accessed on 30 March 2020).

16. Terna. Qualità del Servizio di Trasmissione, Valori Minimi e Massimi Convenzionali Della Corrente di Cortocircuito e Della Potenza di Cortocircuito Della Rete Rilevante con Tensione 380-220-150-132 kV; Terna: Rome, Italy, 2017.

(C) 2020 by the authors. Licensee MDPI, Basel, Switzerland. This article is an open access article distributed under the terms and conditions of the Creative Commons Attribution (CC BY) license (http://creativecommons.org/licenses/by/4.0/). 